\title{
Persistence to 5-year hormonal breast cancer therapy: a French national population-based study
}

\author{
Pauline Bosco-Lévy ${ }^{\star 1,2,3}$, Jeremy Jové2 ${ }^{2}$ Philip Robinson ${ }^{2}$, Nicholas Moore ${ }^{1,2,3}$, Annie Fourrier-Réglat ${ }^{1,2,3}$ \\ and Julien Bezin ${ }^{1,2,3}$ \\ ${ }^{1}$ INSERM, U1219, Univ. Bordeaux, Population Health Research Center, Pharmacoepidemiology and Population Impact of Drugs \\ Team, F-33000 Bordeaux, France; ${ }^{2} \mathrm{ClC}$ Bordeaux ClC1401, F-33000 Bordeaux, France and ${ }^{3} \mathrm{CHU}$ de Bordeaux, Department of \\ Medical Pharmacology, F-33000 Bordeaux, France
}

\begin{abstract}
Background: Non-persistence to oral hormonal therapy (HT) in breast cancer (BC) is an emerging health issue, and estimations vary according to the population selected and/or the statistical method applied. This study aimed to estimate non-persistence over 5 years to HT in an unselected sample of women with BC using a French national population-based database and accounting for competing risks.
\end{abstract}

Methods: A retrospective cohort of 600 women initiating a HT between 2006 and 2007 was constituted using a representative sample of the French national healthcare insurance system database. The Cumulative Incidence Function method was used to estimate the probability of first treatment discontinuation of at least 90 days accounting for competing risk of death from any cause over the theoretical 5-year period of treatment.

Results: Thirty one percent of patients who initiated a HT were identified as non-persistent at the fifth year of follow-up. Patients who switched to another HT (HR 3.10,95\% Cl $(2.20 ; 4.36))$ or had metastatic BC (HR 3.07, 95\% Cl $(1.73 ; 5.46))$ were more likely to be non-persistent. Women who initiated aromatase inhibitors as compared with tamoxifen $(\mathrm{HR} 0.62,95 \% \mathrm{Cl}(0.46 ; 0.83)$ ), had administrative registration for $\mathrm{BC}(\mathrm{HR} 0.21,95 \% \mathrm{Cl}(0.13 ; 0.32))$, or had received an adjuvant chemotherapy $(\mathrm{HR} 0.65,95 \% \mathrm{Cl}$ $(0.48 ; 0.89))$ were less likely to discontinue.

Conclusions: The estimate of long-term non-persistence in an unselected sample of women treated in France by oral hormonal therapy is substantial, even accounting for competing risks.

Breast cancer is a major worldwide public health challenge. It is the most frequently diagnosed cancer among women in the world and represents the leading cause of cancer death (International Agency for research on Cancer, 2012); in France the annual standardised incidence rate of breast cancer was 88/100000 and associated mortality was 16/100 000 in 2012 (Binder-Foucard et al, 2013).

Over the last decades, the development of new therapies has led to a significant improvement in the prognosis for women with breast cancer. Among such therapies, hormonal therapy (i.e., tamoxifen and aromatase inhibitors) is currently recommended as the standard treatment for hormone receptor positive breast tumours. Many clinical trials have demonstrated that both tamoxifen and aromatase inhibitors are efficient to reduce breast cancer recurrence and mortality (Early Breast Cancer Trialists' Collaborative Group (EBCTCG), 2005; Dowsett et al, 2010). However, in real life, the optimal effect of hormonal therapy identified through clinical trials may be compromised by women who do not complete the recommended 5-year course of therapy. Available real-life studies designed to estimate non-persistance have been summarised in a qualitative systematic review (Murphy et al, 2012) from which a

*Correspondence: Dr P Bosco-Lévy; E-mail: pauline.bosco-levy@u-bordeaux.fr

Received 9 March 2016; revised 16 June 2016; accepted 9 August 2016; published online 6 September 2016

(c) 2016 Cancer Research UK. All rights reserved 0007-0920/16 
meta-regression analysis (Huiart et al, 2013) found that between 12 and $14 \%$ of women discontinued their treatement over the first year of treatment, and between 31 and $47 \%$ of them have discontinued at the end of year 5 . These studies were performed using different data sources which may be a major source of variability. For instance, studies based on patient self-report (Fink et al, 2004; Lash et al, 2006; Güth et al, 2012; Stirratt et al, 2015) are exposed to bias such as social desirability response bias and memory bias which may significantly affect the non-persistence estimate (Farmer, 1999). In longitudinal studies based on claims databases, survival models commonly used to describe persistence do not always account for competing risks between events such as treatment discontinuation and death (van Herk-Sukel et al, 2010; Hershman et al, 2011; Huiart et al, 2011, 2012; Nekhlyudov et al, 2011; Neugut et al, 2011). Treating these events as censored observations may lead to a violation of the assumption that censoring and time-to-event distribution are independent and may lead to an overestimation of the non-persistent estimate (Gooley et al, 1999; Satagopan et al, 2004; Southern et al, 2006). In addition, some of these longitudinal claims data based studies are conducted on specific groups of patients (i.e., early stage of breast cancer, elderly and/or lowincome or premonauposal women) (Owusu et al, 2008; Kimmick et al, 2009; van Herk-Sukel et al, 2010; Nekhlyudov et al, 2011; Neugut et al, 2011; Huiart et al, 2012; Weaver et al, 2013), which may limit the generalisation of findings. At last, persistence estimates may vary according to country specific culture and healthcare system, and to our knowledge, no study has examined non-persistence in a representative sample of the French population.

In light of this, it seems essential to estimate non-persistence accounting for competing risks, and using data of an unselected population. The objective of the present study was therefore to assess non-persistance to tamoxifene and aromatase inhibitors (AI) by using Cumulative Incidence Function (CIF) method to account for competing risks among a representative population of women in France over 5 years using the permanent sample of the national healthcare reimbursement database covering the overall French population (66 million individuals in 2015).

\section{MATERIALS AND METHODS}

Data source. This cohort study was conducted using reimbursement data from the Echantillon Généraliste de Bénéficiaires (EGB), a representative $1 / 97$ th dynamic random sample of the national healthcare system population-based database that contains data for 670000 subjects (Roquefeuil et al, 2009; Tuppin et al, 2010). The EGB is an anonymised reimbursement database built by a random selection of individual identification numbers representative of the French population by age and by gender. The EGB includes insured persons, whether they are receiving healthcare or not; all health insurance schemes are included, except some rare special insurance schemes. It contains basic demographic data such as gender and dates of birth or death, all outpatient healthcare reimbursements including characteristics of the prescriber, date of reimbursement, drug dispensations motivating the reimbursement and, for each of these, the dose and the quantity of delivery units (e.g., tablets) dispensed. For each patient, data on affiliation to full healthcare coverage for those on low income (Couverture Médicale Universelle, CMU) and registration with a long-standing disease (Affection Longue Durée, ALD) are available. Patients suffering from one of the 30 recognised long-standing diseases, including cancer, may benefit from ALD status that grants patients copayment exempt status for all medical procedures and services related to these diseases. The general practitioner determines eligibility for ALD status by presenting the patient's medical characteristics to the national health insurance consultant physician who then decides whether or not the patient is eligible for full coverage. The EGB database is linked to a hospital discharge summary database used to assess economic hospital activity (Programme de médicalisation des systèmes d'information, PMSI) that contains hospital data such as diagnoses and medical, surgical, or biological acts. Hospitalisation and ALD medical diagnoses are coded using the International Classification of Diseases, 10th revision (ICD-10). Coding of medical or surgical acts completed during hospitalisations is based on the Common Classification of Medical Acts (CCAM) codes (Supplementary Table).

Study population. Women were eligible for inclusion in the cohort if they were aged 20 years or more and if they initiated a treatment with tamoxifen or AI (i.e., anastrozole, exemestane, or letrozole), between 1 January 2006 and 31 December 2007. Initiation was defined as a first reimbursement without prior reimbursement for one of these drugs during the preceding 12 months. The date of first reimbursement was considered as the index date. Data available within 12 months prior to the first tamoxifen or AI reimbursement were used to identify predictors of non-persistence. The recommended duration of therapy is 5 years (Saint-Paul-de-Vence et al, 2005; Saint-Paul-de-Vence, 2007; Collège de la Haute Autorité de Santé (HAS), 2010) and thus all women selected for the analysis of non-persistence were followed for maximum 5 years after index date, or until death from any cause, or until treatment discontinuation, whichever came first.

Outcome of interest. The outcome measure was non-persistence to hormonal therapy assessed over 5 years after treatment initiation. Treatments delivered during the follow-up were identified using Anatomical Therapeutic Chemical (ATC) codes: exemestane (L02BG06), anastrozole (L02BG03), letrozole (L02BG04), and tamoxifen (L02BA01). Patients were considered non-persistent at the occurrence of the first treatment discontinuation during follow-up. For each woman, the number of days' supply for each reimbursement was calculated using the overall quantity of reimbursed drugs provided by the prescription, divided by the corresponding Defined Daily Dose (DDD). Overlapping days supplied with successive reimbursements for a same treatment were added to the total duration of exposure. However, when women switched from one hormonal therapy to another one, the overlapping days supplied with successive reimbursements were not included in the total duration of exposure.

Discontinuation was defined as a treatment refill gap of 90 days after the estimated date of last treatment day covered by a given reimbursement. To assess variability, a sensitivity analysis was performed using a 30-day gap, 60-day gap, and 120-day gap. Switching from one hormonal therapy to another one was not considered as a treatment gap or discontinuation.

In order to give a measurable value of the non-persistence issue in France in terms of public health, the estimated proportion of non-persistent women was extrapolated to the whole French population. This calculation was performed using multipliers, standardised on age and gender, for each study year, provided by the national health insurance system.

Covariates. Covariates were chosen based on a review of the literature relating to hormonal therapy adherence and persistence (Verbrugghe et al, 2013) and limited to the variables that were available in the EGB.

As a first step, potential predictors of non-persistence were selected at the time of treatment initiation. The specialties of prescribers responsible for therapy initiation were grouped into three categories: general practitioners, breast cancer specialists (radiotherapists, oncologists, gynecologists), or other. Professional activities of prescribers responsible for therapy initiation were categorised as private practitioners, or salaried physicians. To take better account of the treatment exposure during the follow-up 
period, those who switched from one type of hormonal therapy to another one were identified, and the exposure to either type of hormonal treatment was estimated. Thus, 5 treatment groups were identified: tamoxifen only, tamoxifen with switch to AI, AI with switch to tamoxifen, AI only, and multiple switches; the number of switches was categorised as none, 1 , or $\geqslant 2$. The exposure to a hormonal treatment was estimated by combining each day of the follow-up period covered by one distinct dispensed hormonal treatment (tamoxifen or AI).

Other variables were selected within the 12 -month period prior to index date: type of treated breast cancer (last breast cancer diagnosis according to the main diagnostic code in the PMSI hospitalisation database (Supplementary Table); breast cancer ALD registration, affiliation to $\mathrm{CMU}$ (at least one drug reimbursement covered by CMU), medical and surgical acts specific to breast cancer (curative surgery, reconstructive surgery, radiotherapy, chemotherapy, biopsy, and imagery), breast cancer metastases (liver, bone, breast, or brain).

Statistical analysis. Quantitative variables were described in terms of mean and standard deviation, and qualitative variables were described in terms of proportion, including missing data. The cumulative probability of the first discontinuation of at least 90 days was estimated using the Cumulative Incidence Function (CIF) method, which incorporates competing risks of death from any cause (Fine and Gray, 1999). The CIF method is the sum of the conditional probabilities of getting non-persistent given that individual is alive in each little interval of the follow-up period. It gives the probability of being non-persistent accounting for the competing risk of death. Patient follow-up was censured at 5 years (theoretical duration of treatment) or at the date of death from any cause, whichever came first. The cumulative probability of nonpersistence was also calculated for each duration of treatment gap (30, 60 or 120 days) of the sensitivity analysis. CIF estimates were presented as percentage with $95 \%$ confidence intervals (95\% CI). A Cox proportional hazards model was used to assess the predictors of discontinuation (90-day gap) at 5 years after initiating hormonal therapy, adjusting for age at inclusion, hormonal therapy provided, number of metastases, and social status (CMU affiliation). Number of switches and exposure to tamoxifen or AI were considered as time dependent variables in the regression model. Results are presented as Hazard Ratios (HR) with 95\% CI. As breast cancer stage at treatment initiation may influence the therapeutic strategy, an additional analysis restricted on a sub cohort of non-metastatic women (supposedly in stage I-III) was conducted. All analyses were performed using SAS software version 9.4 (SAS Institute, Inc., Cary, NC, USA).

\section{RESULTS}

Study population. The study cohort identified from the EGB database included 600 women over the age of 20 years, who had a first reimbursement of tamoxifen or AI between 1 January 2006 to 31 December 2007, and the median follow-up was 1826 days. After a standardised extrapolation to the national French population, this cohort corresponded to 79793 women in France initiating a hormonal therapy between 2006 and 2007.

Mean age at therapy initiation was 62.0 (standard deviation: 14.1) years. The majority of women had breast cancer ALD registration $(86.2 \%)$ before initiating hormonal therapy. Threequarters $(71.5 \%)$ had invasive breast cancer, and most had been treated by curative surgery $(71.8 \%)$ within the 12 -month period prior to inclusion. For 419 women $(69.8 \%)$ the first reimbursement for hormonal therapy was for an AI, and among these, 251 (59.9\%) had a first reimbursement for anastrozole. During follow-up, 358 women (59.7\%) used AIs only, 107 (17.9\%) used tamoxifen only, and $135(22.6 \%)$ switched from one treatment to another at least once. Over the study period, $110(18.3 \%)$ women died (all-cause mortality; Table 1).

Non-persistence to hormonal therapy. Considering discontinuation as a treatment refill gap of 90 days, $12.0 \%$ (95\%CI $[9.5 ; 14.8])$ of women were non-persistent by the end of the first year of follow-up and this proportion increased to 30.6\% (95\% CI $(26.6 ; 34.6))$ by the end of the fifth year (Figure 1). Among women who experienced a treatment discontinuation, 153 (65.7\%) did not resume therapy before the end of the follow-up period.

Transposed to the national French level, the proportion of nonpersistent women at 1 year after treatment initiation corresponded to $9,575(95 \%$ CI $(7580 ; 11809))$ and after 5 years to 24417 (95\% CI (21 22527608$)$ ) women in France.

Sensitivity analysis. The results from the sensitivity analysis found that for a 30-day refill gap, the most restrictive definition of the outcome, $21.0 \%$ (95\% CI $(17.7 ; 24.5))$ of women were non-persistent at 1 year and $55.1 \%(95 \%$ CI $(50.5 ; 59.5))$ were nonpersistent at 5 years (Figure $2 \mathrm{~A}$ ), which was significantly greater than that found using a 90-day gap. Applying a 60-day or 120-day gap found no significant difference with results obtained with a 90-day gap: using the 60 -day gap $14.1 \%$ (95\% CI $(11.4 ; 17.1)$ ) of women were non-persistent at 1 year and $37.1 \%$ (95\% CI $(32.9 ; 41.4)$ ) were non-persistent at 5 years (Figure $2 \mathrm{~B})$; using the 120 -day gap $10.9 \%(95 \%$ CI $(8.5 ; 13.6))$ of women were nonpersistent at 1 year of treatment and $28.0 \%(95 \%$ CI $(24.1 ; 31.9))$ were so by the end of the fifth year (Figure 2C). Sensitivity analysis also found that among non-persistent women, 142 (38.1\%) did not resume therapy before the end of the follow-up period after a 30-day treatment gap, $154(56.8 \%)$ after a 60-day treatment gap, and $129(65.6 \%)$ after a 120-day treatment gap.

Predictors of non-persistence to hormonal therapy. Factors significantly associated with non-persistence to hormonal therapy at 5 years of follow-up were investigated using the 90 -day refill gap definition of discontinuation. The factors associated with an increased risk of non-persistence were: being exposed to tamoxifen rather than $\mathrm{AI}(\mathrm{HR}: 1.61,95 \% \mathrm{CI}(1.20 ; 2.17))$, switching once from an AI to tamoxifen or vice versa (HR: $3.10,95 \%$ CI $(2.20 ; 4.36)$ ) which was not further increased when switches occured twice or more, and metastatic breast cancer at treatment initiation which increased with the number of metastases ( 1 metastasis: HR: 3.07, $95 \%$ CI $(1.73 ; 5.46)$; $\geqslant 2$ metastases: HR: 4.25, 95\% CI (2.06; 8.78)). Women were significantly less at risk of non-persistence (i.e., more likely to persist) if, during the year prior to therapy initiation, they had breast cancer ALD (HR: 0.21, 95\% CI (0.13; 0.32)) and at least one session of breast cancer chemotherapy (HR: 0.65, 95\% CI (0.48; 0.89); Table 2).

Analysis restricted on the sub cohort of non-metastatic women. Overall, 564 women had no metastases within the 12 months prior to treatement initiation. Their baseline characteristics were generally similar to those of the full cohort except that a higher proportion of women with no metastasis received a curative surgery before treatment initiation comparing with the full cohort (73.4\% vs 71.8\%; Table 1). For a 90-day refill gap definition, $12.4 \%(95 \%$ CI $(9.8 ; 15.3))$ of women with no metastases were non-persistent at 1 year and $30.8 \%(95 \%$ CI $(26.8 ; 34.9))$ were so at the end of the fifth year. For these women, determinants of non-persistence remained similar to those identified in the full cohort (Table 2).

\section{DISCUSSION}

This study showed that after the first year of treatment, a substantial proportion of women in a representative sample 
Table 1. Socio-demographic and treatment characteristics of women initiating a hormonal therapy, with or without metastases, between 1 January 2006 and 31 December 2007 in EGB database

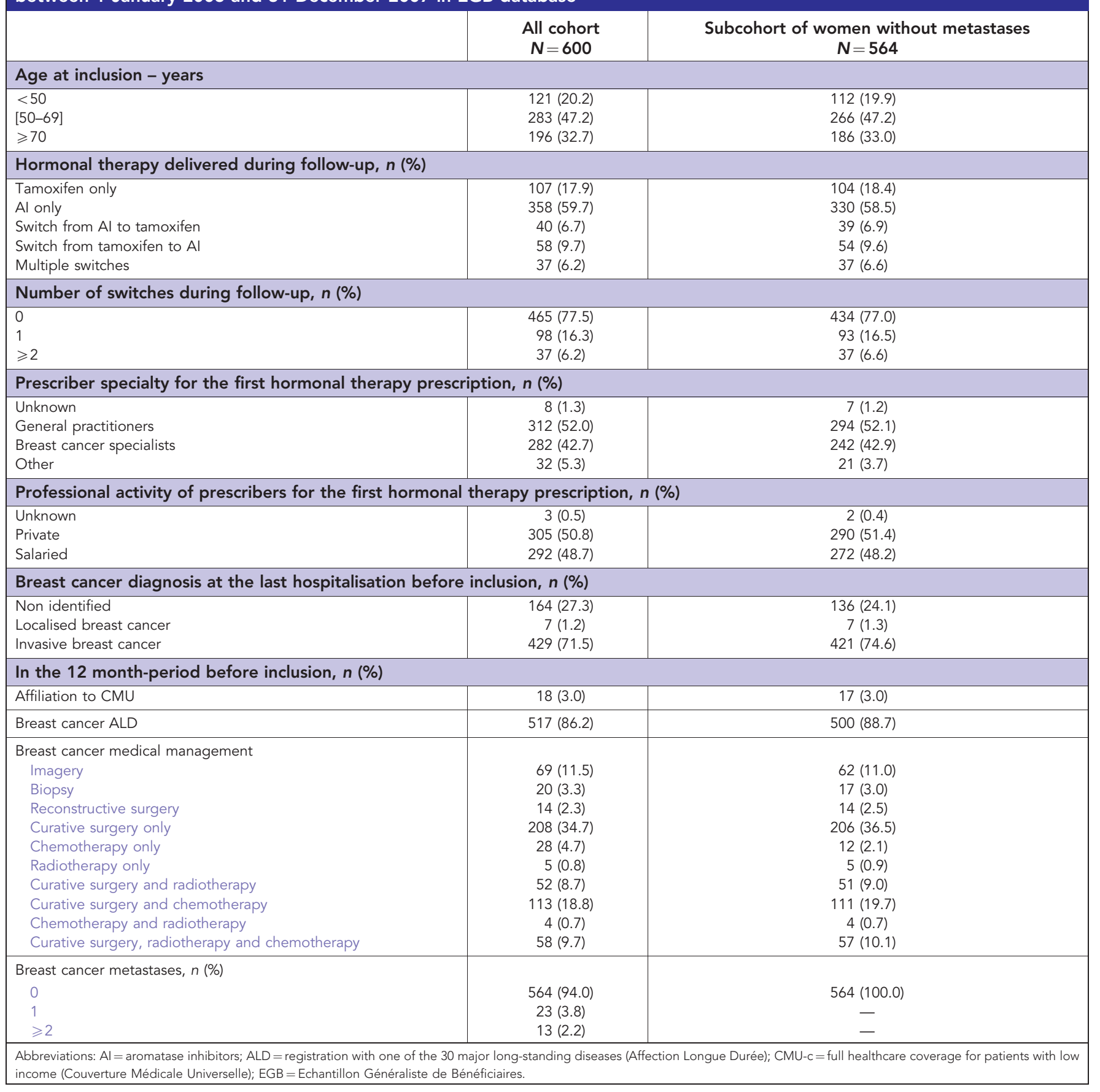

of women treated in France by oral hormonal therapy were non-persistent to their treatment, even when competing risks are accounted for. This proportion tended to increase over the subsequent four years of treatment. The medication-taking behaviour of these patients is all the more important in light of the findings of recent clinical trials that indicate that continuing endocrine therapy for 10 years rather than stopping at 5 years provides an additional overall and disease-free survival benefit (Davies et al, 2013; Smith et al, 2014).

Overall, these estimates were mostly lower than those obtained from healthcare database studies using the same definition of nonpersistence as that used in the present study (Kimmick et al, 2009; Huiart et al, 2011; Nekhlyudov et al, 2011; Weaver et al, 2013). For instance, studies reported by Nekhlyudov et al (2011), Weaver et al
(2013) and Kimmick et al (2009) found that between 14\% and 20\% of women treated by hormonal therapy discontinued treatment for more than 90 days at the end of the first year and that between $46 \%$ and $60 \%$ of them discontinued treatment at the end of the fifth year. One aspect that may explain this apparent inconsistency is that the probabilities of non-persistence were overestimated by the Kaplan-Meier survival analysis that did not account for competing risks (Huiart et al, 2014). Furthermore, these studies focused on specific subgroups of patients; for instance in studies reported by Weaver et al (2013) and Kimmick et al (2009), the study populations included low-income patients covered by Medicaid who are known to be more likely to discontinue their treatment (Streeter et al, 2011). Likewise, in the study reported by Nekhlyudov et al (2011), authors focused on a specific population 
of patients with early-stage breast cancer, and the population was also younger than that of the present study ( $60 \%$ of patients were under 60 years of age). Conversely, our study is population based using data from the French publicly funded health system which

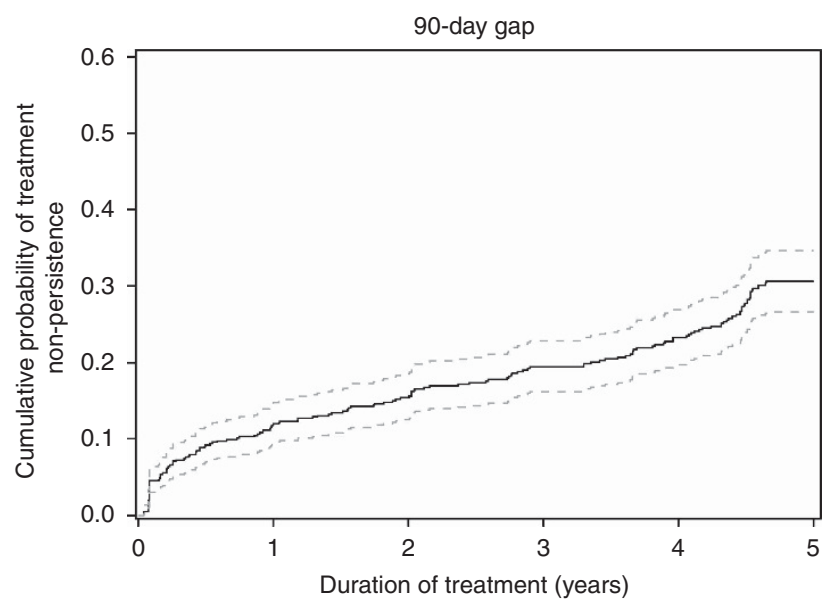

Figure 1. Cumulative probability of non-persistence (continuous line) with $95 \%$ confidence interval (dotted lines) estimated by the Cumulative Incidence Function method during the 5 years following hormonal therapy initiation using a 90-day gap as definition of non-persistence. The plateau observed at the end of the follow up is an artifact that is in relation to the 90-day gap retained to define non-persistence. provides universal coverage to $99.9 \%$ of the French population and thus does not focus on any specific group (Roquefeuil et al, 2009; Tuppin et al, 2010). In contrast, the results of this study are consistent with those of a large population-based cohort of women with breast cancer conducted using the United Kingdom General Practice Research Database, which found that treatment discontinuation reached $29.8 \%$ of patients at 5 years (Huiart et al, 2011).

Among previous studies which have studied persistence in patients treated by hormonal therapy, the minimum treatment gap allowable to remain persistent ranged from 45 to 180 days (Murphy et al, 2012). The choice of a 90-day period to define a gap herein is based on the clinical practice in France where a prescription covers at least 30 days and at most 60 days. A discontinuation of 90 days of treatment means that the patient may have missed at least one visit to the physician to renew prescription. Because the use of a unique duration to define a gap may limit understanding about the true pattern of hormonal therapy use, a sensitivity analysis was conducted using shorter and a longer interval of discontinuation. This found no significant difference in estimates using a 60-day or a 120-day gap compared with that found using a 90-day gap, and, as expected, a significant difference using a 30-day gap which is too sensitive to estimate correctly non-persistence in this present study. These results confirm that a 90 -day period of treatment discontinuation is robust to assess non-persistence to hormonal therapy in this study.

To further understand non-persistence among these women, the present study also highlights some predictors of discontinuation. Among therapy-related factors, it was found that being exposed to
A

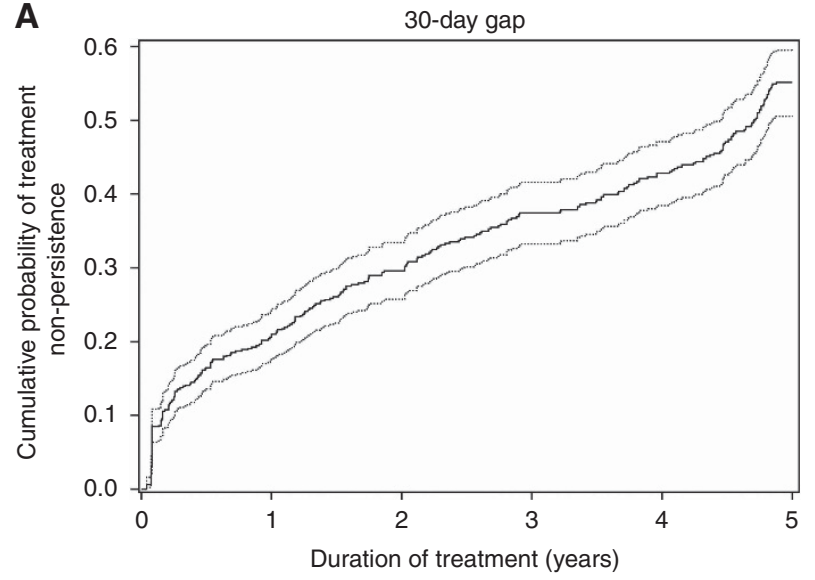

B

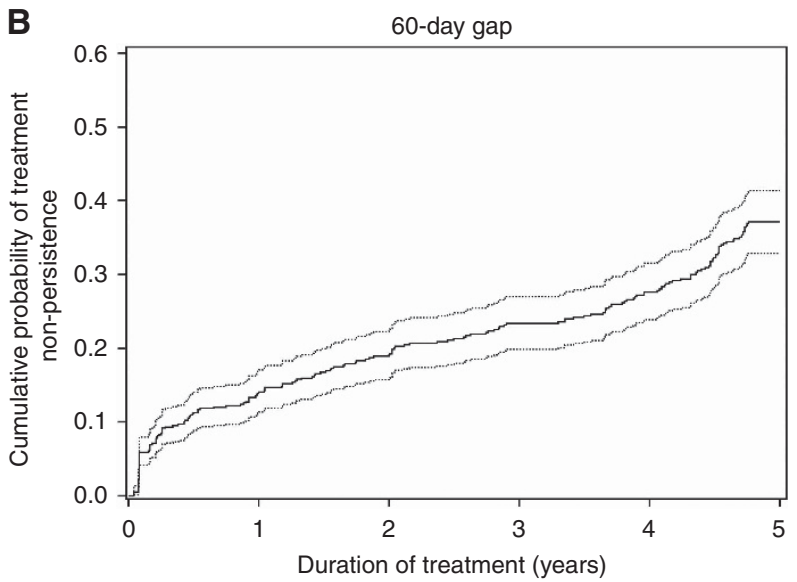

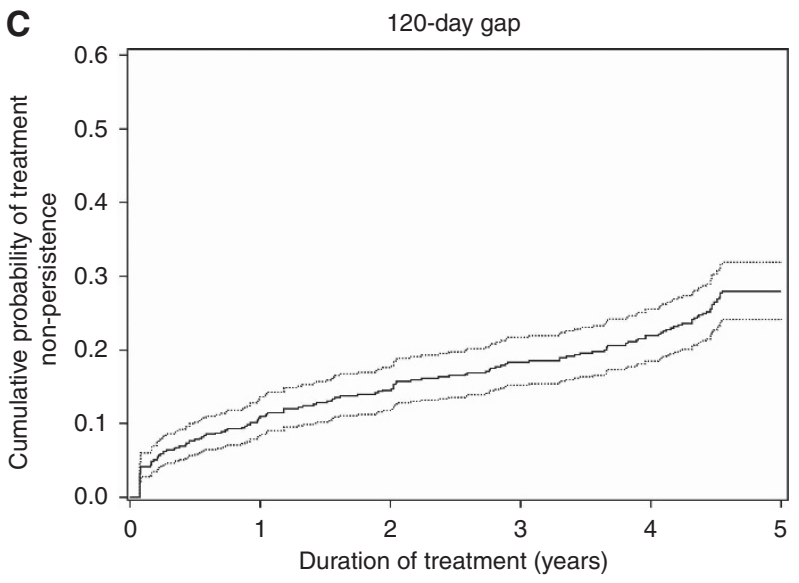

Figure 2. Cumulative probability of non-persistence curves (continuous line) with $95 \%$ confidence interval (dotted lines) estimated by the Cumulative Incidence Function method during the 5 years following the hormonal therapy initiation for each duration of gaps: a 30-day gap (A), a 60-day gap (B), and a 120-day gap (C). The plateau observed for each curve at the end of the follow up is an artifact that is in relation to the duration of gap retained to define non-persistence. 
Table 2. Factors associated with non-persistence (defined as a gap of 90 days) at 5 years of follow-up among women on hormonal therapy with or without metastases, in EGB database, according to multivariate Cox analysis

\begin{tabular}{|c|c|c|c|c|}
\hline & \multicolumn{2}{|l|}{$\begin{array}{l}\text { All cohort } \\
N=600\end{array}$} & \multicolumn{2}{|c|}{$\begin{array}{l}\text { Subcohort of women without metastases } \\
\qquad N=564\end{array}$} \\
\hline & $\begin{array}{l}\text { Adjusted hazard ratio } \\
\qquad(95 \% \mathrm{Cl})\end{array}$ & $P$-value & $\begin{array}{l}\text { Adjusted hazard ratio } \\
(95 \% \mathrm{Cl})\end{array}$ & $P$-value \\
\hline Age - years & & 0.436 & & 0.255 \\
\hline $\begin{array}{l}(50-69) \text { vs }<50 \\
\geqslant 70 \text { vs }<50\end{array}$ & $\begin{array}{l}0.84(0.59-1.19) \\
0.99(0.66-1.50)\end{array}$ & & $\begin{array}{l}0.75(0.52-1.10) \\
0.92(0.61-1.40)\end{array}$ & \\
\hline \multicolumn{5}{|l|}{ Number of switches ${ }^{a}$} \\
\hline $\begin{array}{l}1 \text { vs } 0 \\
\geqslant 2 \text { vs } 0 \text { or } 1\end{array}$ & $\begin{array}{l}3.10(2.20-4.36) \\
2.48(1.39-4.44)\end{array}$ & & $\begin{array}{l}3.49(2.45-4.96) \\
2.65(1.48-4.76)\end{array}$ & \\
\hline \multicolumn{5}{|c|}{ In the 12-month period before inclusion } \\
\hline $\begin{array}{l}\text { Affiliation to CMU-c } \\
\text { Yes vs No }\end{array}$ & $1.18(0.60-2.32)$ & 0.639 & $1.02(0.50-2.09)$ & 0.959 \\
\hline $\begin{array}{l}\text { Breast cancer metastases } \\
\quad 1 \text { vs } 0 \\
\geqslant 2 \text { vs } 0\end{array}$ & $\begin{array}{l}3.07(1.73-5.46) \\
4.25(2.06-8.78)\end{array}$ & $<0.001$ & - & - \\
\hline
\end{tabular}

tamoxifen rather than to $\mathrm{AI}$ and the occurrence of at least one switch from a hormonal therapy to another during the treatment period increased significantly the risk of being non-persistent. This finding may be related to therapy-related side effects as reported in others studies. For instance, in a meta-analysis women who reported side effects were significantly more likely not to persist with hormonal therapy (OR: 5.73, 95\% CI $(3.87 ; 8.47), P<0.001)$ (Cahir et al, 2015). The main reported adverse effects are related to AI (bone loss and arthralgia) for which effective options are currently available to help patients cope with unwanted symptoms (Monnier, 2007; Dent et al, 2011) and continue their treatment. However, although side effects related to tamoxifen, namely, thromboembolic disease and endometrial cancer, are less frequent, they are more severe and difficult to manage, and their occurrence increases with long-term treatment. Moreover, switching from a treatment to another may be an indicator of unmanaged adverse effects; this is illustrated by Guth et al who found that the majority of women who experienced therapy-related side effects switched hormonal therapy (Güth et al, 2011). The results of the present study are in line with that reported by $\mathrm{He}$ et al, who found that patients who switched hormonal therapy during the first year of follow-up were also at increased risk of discontinuation in the following four years (HR: 1.50, 95\% CI (1.23; 1.83); He et al, 2015). Treatment switch may also reflect a lack of treatment efficacy and a progression of the disease, which may lead to a treatment discontinuation initiated by the prescriber.

Among patient clinical factors, age does not appear to be a predictor of non-persistence. This contrasts with results of others studies, which found that extreme ages (i.e., older or younger) were negatively associated with non-persistence (Owusu et al, 2008; Hershman et al, 2010). Concerning disease-related factors, it was found that women with metastatic breast cancer before treatment initiation were more likely to discontinue treatment. At this stage of the disease benefit of endocrine therapy may be low especially considering toxicity of these drugs. Discontinuation of treatment may be a prescriber decision or the choice of patients who might not expect any significant improvement from the hormonal therapy and who want to avoid the toxicity of the treatment, considering it of low benefit. This ties in with that reported by Fink et al (2004), who have previously identified that lack of belief in the efficacy of hormonal therapy was associated with hormonal therapy non-persistence. By contrast, women who received an adjuvant chemotherapy for breast cancer were likely to be persistent as previously reported by several other studies (Fink et al, 2004; Lash et al, 2006; Kimmick et al, 2009; Hershman et al, 2010).

The medical-social-related predictor of persistence that was identified is the ALD registration status before treatment initiation. According to the findings of the present study, women who have this ALD registration were more likely to persist. The ALD system is specific to the French healthcare system and its eligibility is based only on medical criteria. Women who benefit from an ALD registration have a better follow-up then those who do not because these women need to visit their general practitioner to submit an application to the national health insurance consultant physician. It could be surprising that patients would not want to benefit from the ALD status, but for some the recognition of breast cancer as long-standing disease is psychologically difficult and may significantly impact on their social and professional life (Préau et al, 2008).

Additional analyses showed that women supposedly in early BC stage (i.e., with no metastasis) at treatment initiation had similar estimate and predictors of non-persistence than those assessed in the full cohort. Similar considerations than those described above may thus be applicable to these women.

The present study has several important strengths. It relies on a high-quality database, the EGB, which is widely used to study patterns of drug use. The EGB is a dynamic and representative sample of $1 / 97$ th of the French national healthcare system population-based database which provides a coverage of pratically the entire population in France. The EGB includes insured persons, whether they are receiving healthcare or not. All health insurance schemes are included, except some rare special insurance schemes. 
Thus EGB gives an representative overview of healthcare consumption of patients treated with hormonal therapy in a 'real-world' practice and it is well suited to study disease with high prevalence such as breast cancer (Martin-Latry and Bégaud, 2010; Moulis et al, 2015). As for any other healthcare databases, it also avoids reporting bias associated with self-reported drug use. There are, however, certain limitations. These mainly concern those common to most healthcare databases, namely the assumption that treatment dispensation equates to intake, but these databases have been frequently used and validated for the assessment of medication-taking behaviour (Sikka et al, 2005; Andrade et al, 2006). Furthermore, there is limited detailed clinical information regarding comorbidities (e.g., smoking and nutritional status), paraclinical examination results and the exact reason for discontinuation. Thus, it was difficult with electronic medical data, to reliably identify situations where the discontinuation or switch of therapy was mandatory, such as as breast cancer recurrence, treatment toxicity (deep veinous thrombosis or endometrial cancer), or other medical reasons not related to breast cancer (palliative treatment of other malignant disease, pregnancy, etc.). In some of these situations, non-persistence may reflect appropriate care. In some others, patient willingness to stop therapy may be justified by a medical reason such as a desired future fertility (Llarena et al, 2015). But in any of these cases, it remains very complex to determine the real reason of treatment discontinuation and field surveys are valuable tools to complement these results. Another limitation of this study, is the choice of the duration of the time-window, preceding treatment initiation and where basic data were collected. Some data may thus be incomplete because information was provided before the 12 months prior to treatment initiation.

In summary, the estimate of long-term non-persistence measured in an unselected sample of women treated in France by oral hormonal therapy, is substantial in the first year of therapy and rose in subsequent years. These results suggest the need for early detection of non-persistence and intervention to assist in resumption of therapy.

\section{CONFLICT OF INTEREST}

Pauline Bosco-Lévy, Dr A Fourrier-Réglat and Dr J Bezin have nothing to disclose. J Jové, P Robinson and Professor N Moore report that the Pharmacoepidemiology unit has received research funding and grants that have contributed indiscriminately to the salaries of its employees, notably from: Abott, Aptalis, AstraZeneca, Bayer, Baxter, BMS, Boehringer, Erempharma, Helsinn, Leo pharma, Lilly, Lunbeck, Pierre Fabre, Pfizer, Merck Serono, Novartis, Nycomed, Sanofi, Stallergenes, Vifor, Vivatech, from null, outside the submitted work.

\section{REFERENCES}

Andrade SE, Kahler KH, Frech F, Chan KA (2006) Methods for evaluation of medication adherence and persistence using automated databases. Pharmacoepidemiol Drug Saf 15: 565-574.

Binder-Foucard Florence, Belot Aurélien, Delafosse Patricia, Remontet Laurent, Woronoff Anne-Sophie, Bossard Nadine (2013) Estimation nationale de l'incidence et de la mortalite par cancer en France entre 1980 et 2012 (Réseau Francim, Service de biostatistique des Hospices civils de Lyon, Institut de veille sanitaire, Institut national du cancer).

Cahir C, Guinan E, Dombrowski SU, Sharp L, Bennett K (2015) Identifying the determinants of adjuvant hormonal therapy medication taking behaviour in women with stages I-III breast cancer: a systematic review and meta-analysis. Patient Educ Couns pii: S0738-3991(15)00234-7.

Collège de la Haute Autorité de Santé (HAS) (2010) Tumeur maligne, affection maligne du tissu lymphatique ou hématopoiétique cancer du sein (HAS).
Davies C, Pan H, Godwin J, Gray R, Arriagada R, Raina V, Abraham M, Alencar VHM, Badran A, Bonfill X, Bradbury J, Clarke M, Collins R, Davis SR, Delmestri A, Forbes JF, Haddad P, Hou M-F, Inbar M, Khaled H, Kielanowska J, Kwan W-H, Mathew BS, Mittra I, Müller B, Nicolucci A, Peralta O, Pernas F, Petruzelka L, Pienkowski T, Radhika R, Rajan B, Rubach MT, Tort S, Urrútia G, Valentini M, Wang Y, Peto R (2013) Long-term effects of continuing adjuvant tamoxifen to 10 years versus stopping at 5 years after diagnosis of oestrogen receptor-positive breast cancer: ATLAS, a randomised trial. Lancet 381: 805-816.

Dent SF, Gaspo R, Kissner M, Pritchard KI (2011) Aromatase inhibitor therapy: toxicities and management strategies in the treatment of postmenopausal women with hormone-sensitive early breast cancer. Breast Cancer Res Treat 126: 295-310.

Dowsett M, Cuzick J, Ingle J, Coates A, Forbes J, Bliss J, Buyse M, Baum M, Buzdar A, Colleoni M, Coombes C, Snowdon C, Gnant M, Jakesz R, Kaufmann M, Boccardo F, Godwin J, Davies C, Peto R (2010) Metaanalysis of breast cancer outcomes in adjuvant trials of aromatase inhibitors versus tamoxifen. J Clin Oncol 28: 509-518.

Early Breast Cancer Trialists' Collaborative Group (EBCTCG) (2005) Effects of chemotherapy and hormonal therapy for early breast cancer on recurrence and 15-year survival: an overview of the randomised trials. Lancet 365: 1687-1717.

Farmer KC (1999) Methods for measuring and monitoring medication regimen adherence in clinical trials and clinical practice. Clin Ther 21: 1074-1090.

Fine JP, Gray RJ (1999) A proportional hazards model for the subdistribution of a competing risk. J Am Stat Assoc 94: 496-509.

Fink AK, Gurwitz J, Rakowski W, Guadagnoli E, Silliman RA (2004) Patient beliefs and tamoxifen discontinuance in older women with estrogen receptor-positive breast cancer. J Clin Oncol 22: 3309-3315.

Gooley TA, Leisenring W, Crowley J, Storer BE (1999) Estimation of failure probabilities in the presence of competing risks: new representations of old estimators. Stat Med 18: 695-706.

Güth U, Myrick ME, Kilic N, Eppenberger-Castori S, Schmid SM (2012) Compliance and persistence of endocrine adjuvant breast cancer therapy. Breast Cancer Res Treat 131: 491-499.

Güth U, Myrick ME, Schötzau A, Kilic N, Schmid SM (2011) Drug switch because of treatment-related adverse side effects in endocrine adjuvant breast cancer therapy: how often and how often does it work? Breast Cancer Res Treat 129: 799-807.

Hershman DL, Kushi LH, Shao T, Buono D, Kershenbaum A, Tsai W-Y, Fehrenbacher L, Gomez SL, Miles S, Neugut AI (2010) Early discontinuation and nonadherence to adjuvant hormonal therapy in a cohort of 8,769 earlystage breast cancer patients. J Clin Oncol 28: 4120-4128.

Hershman DL, Shao T, Kushi LH, Buono D, Tsai WY, Fehrenbacher L, Kwan M, Gomez SL, Neugut AI (2011) Early discontinuation and nonadherence to adjuvant hormonal therapy are associated with increased mortality in women with breast cancer. Breast Cancer Res Treat 126: 529-537.

He W, Fang F, Varnum C, Eriksson M, Hall P, Czene K (2015) Predictors of discontinuation of adjuvant hormone therapy in patients with breast cancer. J Clin Oncol 33: 2262-2269.

Huiart L, Bouhnik A-D, Rey D, Tarpin C, Cluze C, Bendiane MK, Viens P, Giorgi R (2012) Early discontinuation of tamoxifen intake in younger women with breast cancer: Is it time to rethink the way it is prescribed? Eur J Cancer 48: 1939-1946.

Huiart L, Dell'Aniello S, Suissa S (2011) Use of tamoxifen and aromatase inhibitors in a large population-based cohort of women with breast cancer. Br J Cancer 104: 1558-1563.

Huiart L, Ferdynus C, Dell'Aniello S, Bakiri N, Giorgi R, Suissa S (2014) Measuring persistence to hormonal therapy in patients with breast cancer: accounting for temporary treatment discontinuation. Pharmacoepidemiol Drug Saf 23: 882-889.

Huiart L, Ferdynus C, Giorgi R (2013) A meta-regression analysis of the available data on adherence to adjuvant hormonal therapy in breast cancer: summarizing the data for clinicians. Breast Cancer Res Treat 138: 325-328.

International Agency for research on Cancer (2012) Globocan 2012: Estimated Cancer Incidence, Mortality and Prevalence Worldwide in 2012 (World Health Organization).

Kimmick G, Anderson R, Camacho F, Bhosle M, Hwang W, Balkrishnan R (2009) Adjuvant hormonal therapy use among insured, low-income women with breast cancer. J Clin Oncol 27: 3445-3451. 
Lash TL, Fox MP, Westrup JL, Fink AK, Silliman RA (2006) Adherence to tamoxifen over the five-year course. Breast Cancer Res Treat 99: $215-220$.

Llarena NC, Estevez SL, Tucker SL, Jeruss JS (2015) Impact of fertility concerns on tamoxifen initiation and persistence. J Natl Cancer Inst 107: djv202.

Martin-Latry K, Bégaud B (2010) Pharmacoepidemiological research using French reimbursement databases: yes we can! Pharmacoepidemiol Drug Saf 19: 256-265.

Monnier A (2007) Clinical management of adverse events in adjuvant therapy for hormone-responsive early breast cancer. Ann Oncol 18(Suppl 8): viii36-viii44.

Moulis G, Lapeyre-Mestre M, Palmaro A, Pugnet G, Montastruc J-L, Sailler L (2015) French health insurance databases: what interest for medical research? Rev Med Interne 36: 411-417.

Murphy CC, Bartholomew LK, Carpentier MY, Bluethmann SM, Vernon SW (2012) Adherence to adjuvant hormonal therapy among breast cancer survivors in clinical practice: a systematic review. Breast Cancer Res Treat 134: 459-478.

Nekhlyudov L, Li L, Ross-Degnan D, Wagner AK (2011) Five-year patterns of adjuvant hormonal therapy use, persistence, and adherence among insured women with early-stage breast cancer. Breast Cancer Res Treat 130: $681-689$.

Neugut AI, Subar M, Wilde ET, Stratton S, Brouse CH, Hillyer GC, Grann VR, Hershman DL (2011) Association between prescription co-payment amount and compliance with adjuvant hormonal therapy in women with early-stage breast cancer. J Clin Oncol 29: 2534-2542.

Owusu C, Buist DSM, Field TS, Lash TL, Thwin SS, Geiger AM, Quinn VP, Frost F, Prout M, Yood MU, Wei F, Silliman RA (2008) Predictors of tamoxifen discontinuation among older women with estrogen receptorpositive breast cancer. J Clin Oncol 26: 549-555.

Préau M, Marcellin F, Lert F, Spire B, Moatti J (2008) Rejet ou discrimination de la part de l'entourage en raison de la maladie. In La vie deux ans après le diagnostic de cancer. (Paris), pp 231-341.

Roquefeuil LD, Studer A, Neumann A, Merlière Y (2009) The Echantillon généralistes de bénéficiaires: representativeness, scope and limits. Prat Organ Soins 40: 213-223.

Saint-Paul-de-Vence (2007) Recommandations pour la pratique clinique: "Cancers du sein».

Saint-Paul-de-VenceSaint-Paul-de-Vence PC de, Conseils OLo, Namer C d'organisation: MGligorov J, Luporsi E, Serin D (2005) «Cancers du sein». Oncologie 7: 342-379.
Satagopan JM, Ben-Porat L, Berwick M, Robson M, Kutler D, Auerbach AD (2004) A note on competing risks in survival data analysis. Br J Cancer 91: $1229-1235$.

Sikka R, Xia F, Aubert RE (2005) Estimating medication persistency using administrative claims data. Am J Manag Care 11: 449-457.

Smith IE, Yeo B, Schiavon G (2014) The optimal duration and selection of adjuvant endocrine therapy for breast cancer: how long is enough? Am Soc Clin Oncol Educ Book e16-e24.

Southern DA, Faris PD, Brant R, Galbraith PD, Norris CM, Knudtson ML, Ghali WA. APPROACH Investigators (2006) Kaplan-Meier methods yielded misleading results in competing risk scenarios. J Clin Epidemiol 59: $1110-1114$

Stirratt MJ, Dunbar-Jacob J, Crane HM, Simoni JM, Czajkowski S, Hilliard ME, Aikens JE, Hunter CM, Velligan DI, Huntley K, Ogedegbe G, Rand CS, Schron E, Nilsen WJ (2015) Self-report measures of medication adherence behavior: recommendations on optimal use. Transl Behav Med 5: 470-482.

Streeter SB, Schwartzberg L, Husain N, Johnsrud M (2011) Patient and plan characteristics affecting abandonment of oral oncolytic prescriptions. J Oncol Pract 7: 46s-51s.

Tuppin P, de Roquefeuil L, Weill A, Ricordeau P, Merlière Y (2010) French national health insurance information system and the permanent beneficiaries sample. Rev Epidemiol Sante Publique 58: 286-290.

van Herk-Sukel MP, van de Poll-Franse LV, Voogd AC, Nieuwenhuijzen GA, Coebergh JW, Herings RM (2010) Half of breast cancer patients discontinue tamoxifen and any endocrine treatment before the end of the recommended treatment period of 5 years: a population-based analysis. Breast Cancer Res Treat 122: 843-851.

Verbrugghe M, Verhaeghe S, Lauwaert K, Beeckman D, Van Hecke A (2013) Determinants and associated factors influencing medication adherence and persistence to oral anticancer drugs: a systematic review. Cancer Treat Rev 39: 610-621.

Weaver KE, Camacho F, Hwang W, Anderson R, Kimmick G (2013) Adherence to adjuvant hormonal therapy and its relationship to breast cancer recurrence and survival among low income women. Am J Clin Oncol 36: 181-187.

This work is published under the standard license to publish agreement. After 12 months the work will become freely available and the license terms will switch to a Creative Commons AttributionNonCommercial-Share Alike 4.0 Unported License.

Supplementary Information accompanies this paper on British Journal of Cancer website (http://www.nature.com/bjc) 